\title{
Bassas da India
}

National Cancer Institute

\section{Source}

National Cancer Institute. Bassas da India. NCI Thesaurus. Code C123743.

An atoll in the southern Mozambique Channel that is part of the French Southern and Antartic Lands. 